\title{
Successful Treatment by Fibrin Glue Sealant for Pneumothorax with Chronic GVHD Resistant to Autologous Blood Patch Pleurodesis
}

\author{
Satoshi Iyama ${ }^{1}$, Tsutomu Sato ${ }^{1}$, Kazuyuki Murase $^{1}$, Shohei Kikuchi ${ }^{1}$, Yusuke Kamihara ${ }^{1}$, \\ Kaoru Ono ${ }^{1}$, Kohichi Takada ${ }^{1}$, Koji Miyanishi ${ }^{1}$, Yasushi Sato ${ }^{1}$, Rishu Takimoto ${ }^{1}$, \\ Masayoshi Kobune ${ }^{1}$, Takuro Obama ${ }^{2}$, Masahiro Miyajima², Atsushi Watanabe ${ }^{2}$, \\ Tetsuya Higami ${ }^{2}$, Yasuo Hirayama ${ }^{3}$ and Junji Kato ${ }^{1}$
}

\begin{abstract}
Pneumothorax associated with chronic graft-versus-host disease (cGVHD) after stem cell transplantation is a rare complication. Autologous blood has been used successfully for pleurodesis, which was less toxic than chemical agents. However, when pneumothorax is resistant to pleurodesis, no other procedure is more effective and conservative. Here, we describe a case of myelodysplastic syndromes complicated with cGVHDrelated pneumothorax. His pneumothorax has been resistant to pleurodesis using autologous blood and was treated successfully with fibrin glue sealant. In our limited experience, we believe the best success could be achieved when this method is used to treat persistent pneumothorax with cGVHD.
\end{abstract}

Key words: pneumothorax, chronic GVHD, pleurodesis, autologous blood patch, fibrin glue sealant

(Intern Med 51: 2011-2014, 2012)

(DOI: 10.2169/internalmedicine.51.7355)

\section{Introduction}

Patients with air leak syndrome (ALS) associated with stem cell transplantation, including pneumothorax, mediastinal and subcutaneous emphysema, is one of the complications expected to increase in the future, and frequently have a fatal outcome $(1,2)$.

Persistent air leak is one of the intractable conditions of pneumothorax due to chronic graft-versus-host disease (cGVHD). It may lead to a longer in-hospital stay and impair the quality of life because of the uncomfortable chest drain tube. Toubai et al. reported that when the air leak cannot be treated appropriately, the patient has a very poor prognosis (2).

Pleurodesis might be preferable to surgery for the treatment of pneumothorax with cGVHD for patients with a poor condition. Autologous blood has been used success- fully for pleurodesis (3), which was less toxic and harmful to lung tissue than conventional chemical agents. However, when pneumothorax is resistant to repeated pleurodesis, no other procedure is more effective and conservative.

Here, we present a case of pneumothorax with cGVHD after stem cell transplantation. His pneumothorax has been resistant to pleurodesis using autologous blood and was treated successfully with fibrin glue sealant, which recently has been applied by chest surgeons to stop air leakage from a resected lung surface during an operation. We believe the best success can be achieved when fibrin glue sealant methods are used to treat persistent pneumothorax with cGVHD after stem cell transplantation.

\section{Case Report}

A 21-year-old man, diagnosed with myelodysplastic syndrome (RAEB-2), initially treated with cytosine arabinoside

${ }^{1}$ The Fourth Department of Internal Medicine, Sapporo Medical University School of Medicine, Japan, ${ }^{2}$ Department of Thoracic and Cardiovascular Surgery, Sapporo Medical University School of Medicine, Japan and ${ }^{3}$ Department of Internal Medicine, Higashi Sapporo Hospital, Japan Received for publication January 19, 2012; Accepted for publication April 19, 2012

Correspondence to Dr. Junji Kato, jkato@sapmed.ac.jp 


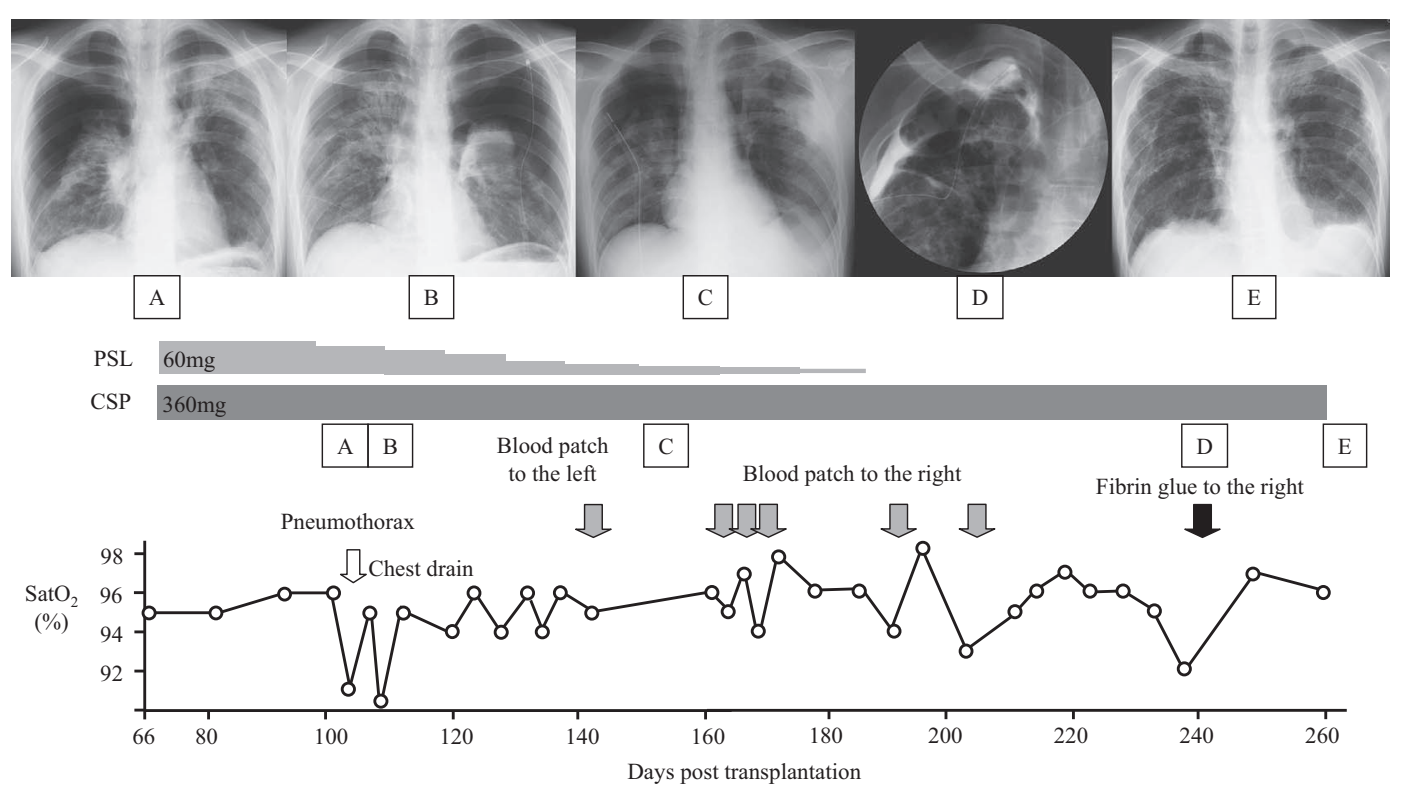

Figure 1. A-C. Chest radiograph showing pneumothorax on both sides. D. Thoracography revealing the location of an air leak. E. Chest radiograph after treatment (day 514).

(100 mg/day for 14 day), underwent allogeneic bone marrow transplantation from his HLA-identical sibling donor after conditioning with $120 \mathrm{mg} / \mathrm{kg}$ of cyclophosphamide and total body irradiation (12 Gy fractionated in 6 doses). Posttransplant GVHD prophylaxis was with $3 \mathrm{mg} / \mathrm{kg} /$ day of cyclosporine (CSP) and short-term methotrexate $\left(10 \mathrm{mg} / \mathrm{m}^{2}\right.$ intravenously on day 1 and $7 \mathrm{mg} / \mathrm{m}^{2}$ intravenously on days 3 , 6). Two months after transplantation he developed acute GVHD involving his skin (grade I), gastrointestinal tract (grade II) and liver (grade III), which was conclusive on biopsy, and then he was given $30 \mathrm{mg}$ of prednisolone (PSL). He subsequently developed severe cGVHD affecting the lung. High resolution CT suggested interstitial lung disease with patchy areas of increased attenuation consistent with mosaic perfusion and bronchial dilatation. These are characteristic findings in bronchiolitis obliterans (BO). Lung function tests confirmed an obstructive and restrictive picture consistent with BO [FEV1.0 40.7\%, \%VC 93.0\%, score 2 (NIH consensus)]. Bronchoalveolar lavage was negative for fungus, Pneumocystis Jirovecii and cytomegalovirus by culture and polymerase chain reaction analysis. He also developed oral cGVHD (xerostomia, score 1), and eyes cGVHD (dry eyes, score 1). Symptoms of cGVHD were controlled by increasing the dose of CSP up to $360 \mathrm{mg}$ and $60 \mathrm{mg}$ of PSL. However, 114 days after transplantation, he developed a productive cough, exertional dyspnea with right-sided chest pain. Chest radiographs demonstrated a large pneumothorax on the right side (Fig. 1A) and showed a newlydeveloped pneumothorax of the left side the next day (Fig. 1B). The insertion of a chest tube and drainage resulted in re-expansion of the right lung (Fig. 1B). Nevertheless, a persistent air leak continued on the left side (Fig. 1B). After 32 days of persistent air leak on the left side, a pleurodesis was attempted using $100 \mathrm{~mL}$ of autologous blood instilled via the chest drain. After a few days, we could remove the chest tube, however, symptoms such as cough, dyspnea and pain recurred two weeks later. Chest radiographs revealed a relapse of pneumothorax of the right side (Fig. 1C). After the insertion of a chest tube, autologous blood pleurodesis was repeated several times to the right side. Nevertheless, the persistent air leak remained refractory to the treatment. Then, a fibrin glue sealant approach was attempted. Briefly, $5 \mathrm{~mL}$ of $400 \mathrm{mg} / \mathrm{mL}$ fibrinogen, $375 \mathrm{U} / \mathrm{mL}$ factor XIII and $5 \mathrm{~mL}$ of $1,250 \mathrm{U} / \mathrm{mL}$ thrombin were drawn into separate syringes that were connected to the ports of the infusion catheter. Under X-ray fluoroscopy, the fibrin glue was infused at the site of the air leak (Fig. 1D). After a few days, the air leak resolved and the tube could be removed (Fig. 1E). The patient has now remained free of any air-leak problems for 10 months.

\section{Discussion}

Recently, Sakai et al. performed a multicenter retrospective study and identified ALS in 18 out of 1,515 recipients (1.2\%) following SCT (1). They reported that cGVHD, second or subsequent SCT, male sex, age $<38$ years and FKbased GVHD prophylaxis were independent risk factors for ALS. Shin et al. reported that multiple factors, including TBI as a conditioning regimen and cGVHD after transplantation, especially from an unrelated donor, may be risk factors for the onset of pneumothorax combined with BO (4). Several reports have indicated that ALS following SCT is associated with severe cGVHD or noninfectious pulmonary complications (5-8), likely to be caused by $\mathrm{BO}$ as in the present case.

Whether an air leak is caused by spontaneous pneumothorax or GVHD -related BO, it can be managed conservatively with a chest drain left in situ and connected to a Heimlich valve (9), or by the injection of irritant agents into the pleu- 
ral cavity, such as tetracycline, quinacrine, talc, or silver nitrate (10). Pleurodesis by the infusion of chemical agents via a chest tube is preferable to surgery for the treatment of pneumothorax when patients are in a poor condition. Also in the present case, since surgical options requiring general anesthetics were considered to be high risk due to the severity of the patient's underlying lung condition, pleurodesis seemed preferable. However, chemical agents used for pleurodesis have serious documented risks such as tissue injury and irreversible damage. Thus, we decided to use autologous blood for pleurodesis.

Autologous blood has been used for pleurodesis for a number of years when spontaneous pneumothorax is recurrent and chronic (11-13). The pathophysiological mechanism mainly responsible for the sealing effect of blood is an inflammatory reaction in the pleural cavity that may contribute to the adhesion between the parietal and visceral pleurae like chemical agents. Further, it is likely that blood directly covers the lacerations in the visceral pleura and pulmonary parenchyma and seals the air leak (11). However the selection of first line therapy of pneumothorax should be judged according to the severity of disease, the previous report by Chadwick et al. that autologous blood patch has been successfully used for pneumothorax complicating cGVHDrelated BO (14) encouraged us to select autologous blood.

In previous studies, different volumes of autologous blood were instilled via thoracostomy tubes, ranging from 50 to $250 \mathrm{~mL}$, given on one to three occasions. It has been recommended that autologous blood should be given as few times as required to obtain the desired result (10). In the present case, a volume of $100 \mathrm{~mL}$ was used with no problems, and one procedure was already effective on the leftside pneumothorax. However, repetition treatment several times was not effective on the right-side pneumothorax. This was likely to have been due to low platelet counts and the low hematocrit of our patient. Furthermore, one of the reasons for the failure may have been because the lacerations in the visceral pleura and pulmonary parenchyma were so large that autologous blood could not cover and seal the air leak. It may have been better to use a fibrin glue method earlier because the therapeutic effect of autologous blood pleurodesis for our patient was not complete.

Recently chest surgeons have often applied fibrin glue to stop air leakage from a resected lung surface. Kinoshita et al. reported that when the fibrin glue is applied to a pneumothorax patient with an unexpanded lung, it is expected that the fibrin glue will first cover the air leak, subsequently produce lung expansion (15). Then, the fibrin glue sealant method was applied to the right side of the lung, which was resistant to repeated blood pleurodesis. The result has been fully satisfactory as described in "Case Report" section. Recently, Thistlethwaite et al. proposed this approach with fibrin glue for sealing the air leak of spontaneous pneumothorax (3). In their report, the air leak bubbling stopped or was greatly diminished in all patients. They used the fibrin sealant mixture containing $1,000 \mathrm{U} / \mathrm{mL}$ thrombin. Thus, here we used BOLHEAL (Teijin Pharma, Japan) $5 \mathrm{~mL}$ containing $1,250 \mathrm{U} / \mathrm{mL}$ thrombin. The advantage of this approach is direct visualization of the procedure under X-ray fluoroscopy and targeted sealing of the air leak without damaging healthy lung. However, the treatment failed because the fibrin often clotted inside the drain. To overcome these problems, Kinoshita et al. diluted the fibrin glue, allowing it to spread more easily from the drain tube into the pleural cavity, and also added contrast medium to facilitate checking by X-ray fluoroscopy (15).

Since the fibrin glue sealant method was dramatically effective in the present case, we strongly recommend this procedure for the treatment of intractable pneumothorax due to cGVHD-related BO. In addition, the fibrin sealant which we have used in this case was not autologous. We used a conventional fibrin sealant which includes human plasma and bovine-derived components. Thus, this fibrin sealant has the potential infective and antigenetic risk. In the future, it may be recommended to use a medical device for the preparation of an autologous fibrin sealant from the patient's blood, such as the Vivostat system (Vivostat A/S, Alleroed, Denmark) (16).

Although this is the first report demonstrating the efficacy of the fibrin glue sealant method for pneumothorax due to cGVHD-related BO, we dare say that this method might be preferable to pleurodesis with autologous blood because pleurodesis is known to decrease respiratory function due to the adhesion of lung with the thoracic wall. In particular, it may become more severe and invasive for patients with lung cGVHD. On the one hand, the fibrin glue sealant method targets the visceral pleura and does not rely on the adhesion of lung to the thoracic wall to prevent recurrence (17). In addition, repeated autologous blood patches may increase the risk of bacterial contamination (18). In the future, it may become possible to avoid pleurodesis, considering the currently emerging interventional approaches, such as the fibrin glue sealant technique described here.

In conclusion, we believe that the best success could be achieved when the fibrin glue sealant method is used to treat persistent pneumothorax with cGVHD after stem cell transplantation.

The authors state that they have no Conflict of Interest (COI).

\section{References}

1. Sakai R, Kanamori H, Nakaseko C, et al. Air-leak syndrome following allo-SCT in adult patients: report from the Kanto Study Group for Cell Therapy in Japan. Bone Marrow Transplant 46: 379-384, 2010

2. Toubai T, Tanaka J, Kobayashi N, et al. Mediastinal emphysema and bilateral pneumothoraces with chronic GVHD in patients after allogeneic stem cell transplantation. Bone Marrow Transplant 33: 1159-1163, 2004.

3. Thistlethwaite PA, Luketich JD, Ferson PF, Jamieson SW. Ablation of persistent air leaks after thoracic procedures with fibrin sealant. Ann Thorac Surg 67: 575-577, 1999. 
4. Shin HJ, Park CY, Park YH, et al. Spontaneous pneumothorax developed in patients with bronchiolitis obliterans after unrelated hematopoietic stem cell transplantation: Case report and review of the literature. Int J Hematol 79: 298-302, 2004.

5. Kumar S, Tefferi A. Spontaneous pneumomediastinum and subcutaneous emphysema complicating bronchiolitis obliterans after allogeneic bone marrow transplantation-case report and review of literature. Ann Hematol 80: 430-435, 2001.

6. Vogel M, Brodoefel H, Bethge W, et al. Spontaneous thoracic airleakage syndrome in patients following allogeneic hematopoietic stem cell transplantation: causes, CT-follow up and patient outcome. Eur J Radiol 60: 392-397, 2006.

7. Galanis E, Litzow MR, Tefferi A, Scott JP. Spontaneous pneumomediastinum in a patient with bronchiolitis obliterans after bone marrow transplantation. Bone Marrow Transplant 20: 695 696, 1997.

8. Hoshino Y, Hatake K, Mimuro J, et al. Refractory bilateral pneumothoraces complicated with interstitial pneumonitis after bone marrow transplantation. Jpn J Clin Hematol 34: 718-722, 1993.

9. Ponn RB, Silverman HJ, Frederico JA. Outpatient chest tube management. Ann Thorac Surg 64: 1437-1440, 1997.

10. Rivas de, Andres JJ, Blanco S, De la, Torre M. Postsurgical pleurodesis with autologous blood in patients with persistent air leak. Ann Thorac Surg 70: 270-272, 2000.

11. Dumire R, Crabbe MM, Mapin FG, Fontenelle LJ. Autologous "blood patch" pleurodesis for persistent pulmonary air leak. Chest
101: 64-66, 1992.

12. Robinson CL. Autologous blood for pleurodesis in recurrent and chronic spontaneous pneumothorax. Can J Surg 30: 428-429, 1987.

13. Mallen JK, Landis JN, Frankel KM. Autologous "blood patch" pleurodesis for persistent pulmonary air leak. Chest 103: 326-327, 1993.

14. Chadwick C, Marven SM, Vora AJ. Autologous blood pleurodesis fot pneumothorax complicationg graft-versus-host disease-related bronchiolitis obliterans. Bone Marrow Transplant 33: 451-453, 2004.

15. Kinoshita $\mathrm{T}$, Miyoshi $\mathrm{S}$, Katoh $\mathrm{M}$, et al. Intrapleural administration of a large amount of diluted fibrin glue for intractable pneumothorax. Chest 117: 790-795, 2000.

16. Moser C, Opitz I, Zhai W, et al. Autologous fibrin sealant reduces the incidence of prolonged air leak and duration of chest tube drainage after lung volume reduction surgery: A prospective randomized blinded study. J Thorac Cardiovasc Surg 136: 843-849, 2008.

17. Kurihara M, Kataoka H, Ishikawa A, Endo R. Latest treatments for spontaneous pneumothorax. Gen Thorac Cardiovasc Surg 58: 113-119, 2010.

18. Lang-Lazdunski L, Coonar AS. A prospective study of autologous 'blood patch' pleurodesis for persistent air leak after pulmonary resection. Eur J Cardiothorac Surg 26: 897-900, 2004.

(C) 2012 The Japanese Society of Internal Medicine

http://www.naika.or.jp/imindex.html 